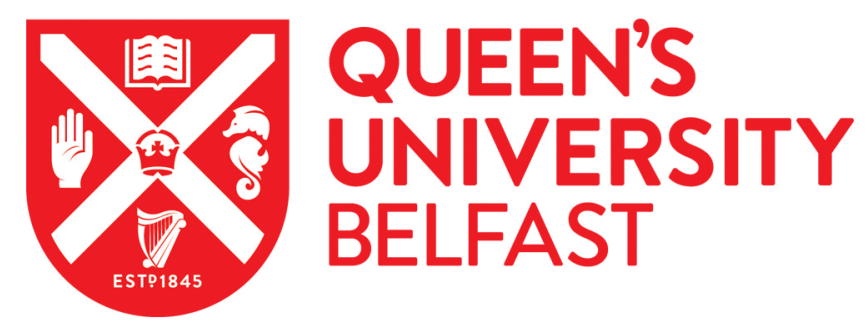

\title{
Microplastic Detection in Soil and Water Using Resonance Microwave Spectroscopy: A Feasibility Study
}

Malyuskin, O. (2020). Microplastic Detection in Soil and Water Using Resonance Microwave Spectroscopy: A Feasibility Study. IEEE Sensors Journal. https://doi.org/10.1109/JSEN.2020.3011311

\author{
Published in: \\ IEEE Sensors Journal
}

Document Version:

Peer reviewed version

Queen's University Belfast - Research Portal:

Link to publication record in Queen's University Belfast Research Portal

\section{Publisher rights}

(C) 2020 IEEE.

This work is made available online in accordance with the publisher's policies. Please refer to any applicable terms of use of the publisher.

\section{General rights}

Copyright for the publications made accessible via the Queen's University Belfast Research Portal is retained by the author(s) and / or other copyright owners and it is a condition of accessing these publications that users recognise and abide by the legal requirements associated with these rights.

Take down policy

The Research Portal is Queen's institutional repository that provides access to Queen's research output. Every effort has been made to ensure that content in the Research Portal does not infringe any person's rights, or applicable UK laws. If you discover content in the Research Portal that you believe breaches copyright or violates any law, please contact openaccess@qub.ac.uk. 


\title{
Microplastic Detection in Soil and Water Using Resonance Microwave Spectroscopy: A Feasibility Study
}

\author{
Oleksandr Malyuskin
}

\begin{abstract}
A feasibility study of microplastic detection and quantification in soil and water using resonance microwave reflectometry is carried out using artificially created samples with a high volumetric concentration of microplastic with $50 \mu \mathrm{m}-0.5 \mathrm{~mm}$ particles size. A mathematical model expressing microplastic concentration in soil and water as a linear function of the measured $S_{11}$ resonance frequency shift and relative permittivity contrast is developed and is found to be in an excellent agreement with the experimental data based on synthetic contaminated material samples. Next, this model is applied to find the best achievable theoretical resolution of microplastic concentration in the natural environment using microwave sensing technology, which is shown to be at around 100ppm (parts-permillion) level in the linear signal detection regime. It is demonstrated that the best achievable level of microplastic contaminant resolution depends on the sensor probe $Q$-factor and sensitivity of the microwave receiver. The bound for the achievable contaminant concentration resolution is found in the analytical form for high-Q resonance microwave sensors of arbitrary geometry. Even though several well-established protocols based on optical, infrared, and X-ray spectroscopy are currently being used for microplastic detection in the natural environment, microwave spectroscopy could offer additional benefits, especially for low-cost, real-time in-situ microplastic detection in diverse environmental conditions outside of the laboratory space.

Index Terms-Sensor phenomena and characterization, sensor systems and applications, microwave antenna, microwave sensor, environmental sensing and monitoring, resonance.
\end{abstract}

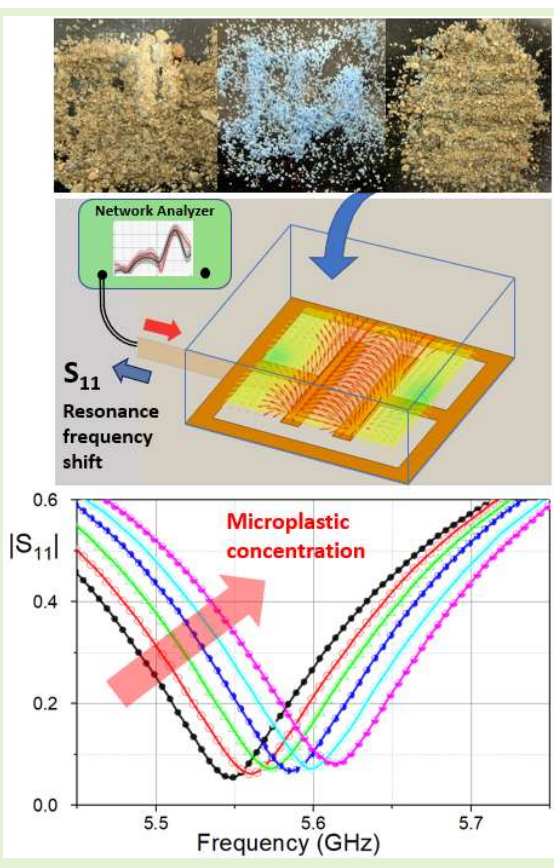

\section{Introduction}

$\mathrm{M}$ ICROPLASTICS are diverse polymer-based particles of characteristic size below $5 \mathrm{~mm}$ that were found in abundance in the natural environment including marine, fresh and drinking water, wastewater, soils, food and air [1]-[5]. Microplastic pollution is a growing environmental problem due to the current scale of global plastic production, estimated as 320 million tons per year [6], insufficient (9\%-14\%) recycling of plastic waste and relatively short life-span of plastic products, half of which are estimated to turn into trash in less than one year [7]. Eventually, more than $80 \%$ of manufactured plastic that has not been recycled ends up in landfills or released into the environment through various pathways [8].

Recent studies [2], [9]-[11] suggest that microplastic pollution poses a significant threat to aquatic life, biochemistry of soils, and could be dangerous to human health through the increased microplastic consumption in food and drinking water. Microplastic detection, quantification and monitoring in the

The paper is submitted for review on.18 April 2020.

O. Malyuskin is with Centre for Wireless Innovation, ECIT, Queen's University Belfast, Queen's Road, NI Science park, BT3 9DT (email:o.malyuskin@qub.ac.uk). natural environment, food and air is an essential part of environmental pollution minimization, our understanding of the microplastic pollution mechanisms, key characteristics of micro- and nano-plastic contaminants and their fate in the environment [12]-[14].

There are several well-established physical protocols for the analysis of environmental microplastics in the $1 \mu \mathrm{m}$ to $5 \mathrm{~mm}$ particle size range [12]-[15]. These include visual inspection, optical microscopy, Fourier-Transform infrared (FTIR) spectroscopy, Raman/micro-Raman spectroscopy, scanning electron microscopy and X-ray photoelectron spectroscopy. In many situations, especially for smaller microplastic particles with a size less than $200 \mu \mathrm{m}$, the visual microscopic analysis of microplastics contamination alone is unreliable, leading to inaccurate estimation of microplastic contamination levels [15].

Infrared (IR), optical and X-ray spectroscopic techniques can be applied for accurate microplastics quantification in the samples with sub-ppm (parts per million) concentration resolution [16]. However, the application of the optical/IR/X- 
ray spectroscopic methods is limited to the dedicated laboratory space due to the high cost and complexity of equipment, complex procedures of calibration and sample preparation, and the necessity in sophisticated software algorithms for spectroscopic data analysis [17].

In this study, we explore the applicability of resonance microwave spectroscopy for the microplastic detection and quantification using artificially created samples of microplastic-contaminated soil and water. The use of artificial samples with precisely-controlled high-level contaminant concentration allows one to quantify the proposed method sensing resolution without the need for cross-calibration by optical, FTIR, or Raman spectroscopic techniques.

Microwave spectroscopic characterization of multicomponent materials such as microplastic-contaminated soil or water, is based on the permittivity contrast between the host medium (uncontaminated soil or water) and the microplastic contaminant. This contrast is high, several times difference, in the microplastic-in-soil and extremely high, more than orderof-magnitude difference, in the microplastic-in-water sensing scenarios, which creates the opportunity for very accurate microplastic detection and quantification at low concentration levels.

Essential features of microwave spectroscopy [18]-[20] include a low cost of microwave sensors, their small size, rugged design for real-time, in-situ operation. These features are attractive for the in-situ microplastic detection and quantification in the flexible sea monitoring platforms [21], sewage and wastewater processing plants [22], smart household sensing devices for detecting microplastics in drinking water and food.

Practical in-situ detection of microplastic in the environment using microwave sensing technology requires the solution of several challenges. First, this method needs a calibration procedure in order to measure the resonance frequency difference between the uncontaminated and microplasticcontaminated material. In the case of a microplastic-in-water sensing scenario, the calibration sample can be straightforwardly obtained in situ by streaming the water flow into parallel mini- or microfluidic channels and using filtration or density separation techniques [23] to obtain the microplasticfree calibration sample. Two identical microwave resonators acting in tandem can be used to measure the relative resonance frequency shift and quantify microplastic contamination level. Apparently, this sensing setup can operate as a self-calibrated sensor.

Sensor calibration procedure is more challenging in the microplastic-in-soil sensing scenario than in microplastic-inwater detection due to a versatile chemical soil composition and soil particle size distribution, moisture content, and ambient temperature variation. In principle, calibration samples can be prepared for the specific soil types using optical, IR or X-ray methods [14], [24]. Microwave detection of microplastic pollution can be used, with some limitations, for these specific soil types only.

The novelty of the present contribution is threefold. First, an analytical closed-form approximation describing the Sparameter resonance frequency shift of a high-Q microwave sensor as a linear function of the contaminant concentration in a two-component material (host medium with a contaminant) is derived from the first principles. This analytical model allows accurate characterization of arbitrary two-component mixtures with low or moderate electromagnetic loss, when the effect of the resonance frequency pulling [25] due to electromagnetic loss in a material is small or negligible as compared to the resonance frequency shift caused by the material permittivity variation. A linear or quasi-linear S-parameter resonance frequency shift as a function of the additive material concentration in the two-component materials was experimentally observed in several recent studies dealing with microwave spectroscopy of polluted water, two-phase liquid mixtures [19], [26]-[29] and dielectric powder materials, [30]. The analytical model derived in this paper explains this phenomenon and can be used for the experimental data analysis in a wide variety of microwave sensing scenarios [31], [32].

Secondly, the system-level criteria establishing the contaminant concentration resolution level are formulated in the explicit form based on the analytical expression for the Sparameter magnitude, frequency tuning resolution and amplitude sensitivity of a typical microwave receiver. This analysis does not depend on a specific microwave sensor geometry and can be applied to any high-Q resonance sensor with S-parameter satisfying a general equation (B1).

Thirdly, the experimental results demonstrating the feasibility of microplastic detection and concentration resolution using microwave spectroscopy are presented for the first time. These experimental results are in good agreement with the analytical model predicting sensor resolution limits based on the S-parameter resonance frequency shift created by the contaminant concentration variation.

Finally, this study discusses the challenges associated with the in-situ environmental microplastic detection using microwave resonance sensing and provides recommendations on optimal microplastic sensing techniques based on the theoretical modeling and experimental results.

\section{THEORETICAL BACKGROUND}

\section{A. Reflectometry principle of operation and microwave sensor characteristics}

A typical microwave reflectometry sensing system, Fig. 1a), consists of a signal source (TX), microwave probe or antenna electromagnetically (EM) interacting with a sample under test, and a receiver (RX) which combines the reflected signal from the probe and a reference signal from the source and generates an interferometric output signal.

The reflected signal from the probe is characterized by the $\mathrm{S}_{11}$ parameter [33] and carries the information about the sample-under-test permittivity. Microwave reflection spectra of the sample are obtained by sweeping the frequency of the source TX and collecting the $S_{11}$ data, from which the material parameters (such as microplastic concentration in the sample) can be extracted using a suitable mathematical model. The two most important parameters of the microwave reflection spectrum are the resonance frequency defined as the frequency where the magnitude of $\mathrm{S}_{11}$ is minimal and Q-factor defined as the ratio of the resonance frequency to the resonance bandwidth at $3 \mathrm{~dB}$ level [33]. 


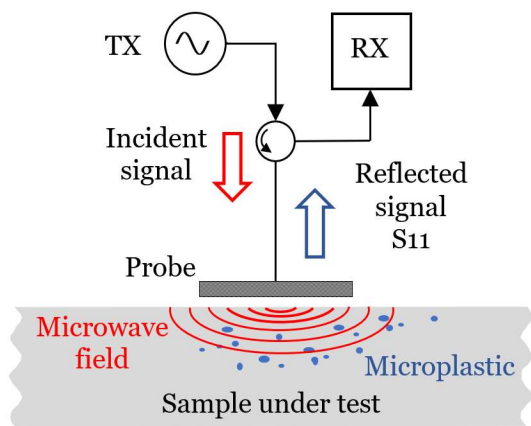

(a)

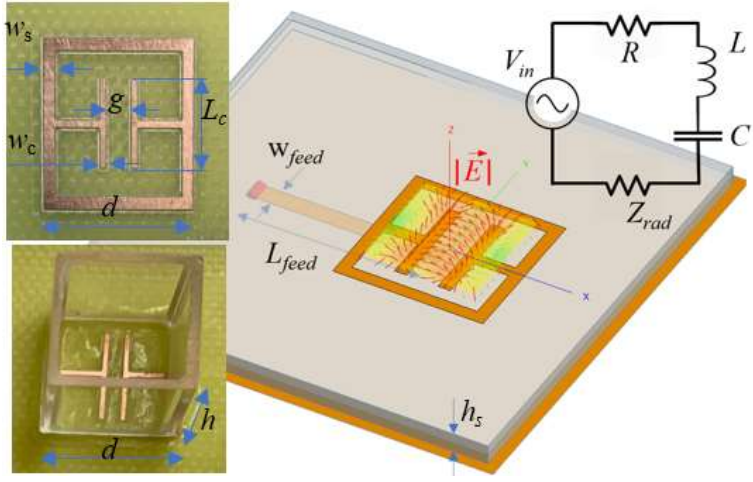

(b)

Fig.1. (a) Schematic diagram of the microwave reflectometry setup. (b) printed sensor geometry and integrated soil sample holder; CAD model, simplified equivalent circuit model (top corner inset) and simulated $E$-field distribution in the proximity to the sensor aperture at $1 \mathrm{~mm}$ stand-off distance.

Microwave probes of various geometries can be used for the considered sensing scenario such as microwave cavities or dielectric resonators [25], [30]-[35], printed sensors including split-ring [36], microstrip [37], [38], loaded apertures or minicoil sensors [18]. In this work, a capacitively-loaded PCB square ring, Fig.1b), also called an electric inductive-capacitive resonator [38], is used for the experimental validation.

TABLE I

SimULATEd PERFoRMANCE DATA OF THE LOADED SQUARE LOOP SENSOR

\begin{tabular}{llllll}
\hline $\begin{array}{c}\mathrm{g} \\
(\mathrm{mm})\end{array}$ & $\begin{array}{c}\text { Resonance } \\
\text { frequency } \\
(\mathrm{GHz})\end{array}$ & Q-factor & $\begin{array}{c}|E| \\
\mathrm{z}=1 \mathrm{~mm} \\
(\mathrm{~V} / \mathrm{m})\end{array}$ & $\begin{array}{c}|E| \\
\mathrm{z}=10 \mathrm{~mm} \\
(\mathrm{mV} / \mathrm{m})\end{array}$ & $\begin{array}{c}\text { Rad. } \\
\text { gain } \\
(\mathrm{dBi})\end{array}$ \\
\hline 0.5 & 5.40 & 223.1 & 0.91 & 88.64 & 3.03 \\
1.0 & 5.57 & 124.6 & 1.00 & 97.35 & 3.08 \\
1.5 & 5.71 & 95.2 & 0.98 & 102.87 & 3.12 \\
2.0 & 5.83 & 87.1 & 0.91 & 105.18 & 3.14 \\
2.5 & 5.98 & 89.6 & 0.80 & 104.74 & 3.16 \\
3.0 & 6.10 & 93.4 & 0.67 & 99.22 & 3.18 \\
\hline \hline
\end{tabular}

The reason for this choice is i) simple, fully planar sensor geometry, ii) $E$-field concentration in the vicinity of the sensor aperture inside the capacitive gap and around the capacitive plates, Fig1b). This field concentration is essential for efficient EM coupling between the sensor and the sample-under-test material; iii) high Q resonance, Q-factor is more than 200, iv) the possibility of the resonance frequency control by a small change in the gap dimensions which can be employed for sensor-array based smart sensing [38]; v) moderate far-field radiation gain, Table I, which can be used for the remote RFIDtype interrogation of the immersed-into-soil sensor response in the in-situ sensing scenario.

Table I summarizes the full-wave simulated EM parameters of the sensor for the dimensions $d=12 \mathrm{~mm}, L_{c}=8 \mathrm{~mm}, \mathrm{w}_{\mathrm{s}}=\mathrm{w}_{\mathrm{c}}$ $=1 \mathrm{~mm}, L_{\text {feed }}=40 \mathrm{~mm}, w_{\text {feed }}=1.5 \mathrm{~mm}$ and gap $\mathrm{g}$ being changed from $0.5 \mathrm{~mm}$ to $3 \mathrm{~mm}$ with $0.5 \mathrm{~mm}$ step. The FR4 substrate thickness $h_{s}$ is $1.6 \mathrm{~mm}$, the feeding strip is vertically separated at $0.8 \mathrm{~mm}$ from the ground plane and the sensor aperture. The input power at the $50 \Omega$ feeding port is $-47 \mathrm{dBm}$. It is important to note that the E-field magnitude exponentially attenuates with the vertical stand-off distance from the gap, therefore sample under test placement and its geometry plays a very important role in the sensing procedure. This is explained in detail in Section III. It should be said that the simulated parameters are in good agreement with the experimental data, Section III.

\section{B. Mathematical model of the $S_{11}$ resonance frequency shift}

The resonance frequency shift $\Delta f=\left|f_{0}-f_{c}\right|$ between the $\mathrm{S}_{11}$ spectral line of the uncontaminated sample (resonance frequency $f_{0}$ ) characterized by the relative permittivity $\varepsilon_{r}^{(h)}$ and the spectral line of the contaminated sample (resonance frequency $f_{c}$ ) with relative permittivity $\varepsilon_{r}^{(m i x)}$,

$$
\varepsilon_{r}^{(m i x)}=V_{h} \varepsilon_{r}^{(h)}+V_{c} \varepsilon_{r}^{(c)}
$$

can be written in the analytical form as (Appendix A)

$$
\Delta f=\frac{1}{2} f_{0} V_{c} \frac{{\varepsilon_{r}^{\prime}}_{r}^{(h)}-\varepsilon_{r}^{\prime(c)}}{A+\varepsilon_{r}^{\prime(h)}}
$$

In (1), $V_{h}$ and $V_{c}$ are the volumetric concentrations (per unit volume) of the host and contaminant material, respectively $V_{h}+V_{c}=1$. Formula (1) describes the relative permittivity of a two-component mixture composed of the host medium and contaminant when $V_{c}<<V_{h}$, [39], [40].

In (2), $\varepsilon_{r}^{\prime(h)}$ and ${\varepsilon^{\prime}}_{r}^{(c)}$ are the real parts of the complex-valued relative permittivity of the host medium and contaminant respectively. $A$ is a constant that depends on the sensor geometry [41] and, in general, the permittivity of the sample, and can be found from comparing the reflection spectra of an empty (unloaded) sensor and a sensor loaded with a calibrated sample under test, (e.g. clean soil). A detailed derivation of (2) is provided in Appendix A. Equation (2) is derived for a highQ sensor with negligible frequency pulling due microwave loss of material [25].

It is important to note that for small contaminant concentrations, $V_{c}<0.1 V_{h}$, the resonance frequency shift $\Delta f$ is linearly proportional to the contaminant concentration $V_{c}$ and the permittivity contrast $\varepsilon_{r}^{\prime(h)}-\varepsilon_{r}^{\prime(c)}$. Since the permittivities of the host medium (e.g. uncontaminated water or soil) and microplastic contaminant can be easily measured or obtained from the reference data, equation (2) provides the analytical basis to quantify microplastic concentrations by measuring the resonance frequency shift of the microwave signal, reflected from the sample under test. 


\section{Theoretical limits of microplastic concentration resolution}

From (2), it can be seen that the microplastic volumetric concentration in the host medium

$$
V_{c}=2 \frac{\Delta f}{f_{0}} \frac{A+\varepsilon^{\prime}{ }_{r}^{(h)}}{{\varepsilon^{\prime}}_{r}^{(h)}-\varepsilon^{\prime(c)}}
$$

is linearly proportional to the relative frequency shift $\Delta f / f_{0}$, for the effective permittivity linear approximation (1).

Quantification of the microplastic concentration in the host material from the $S_{11}$ spectra is based on the detection of the resonance frequency of an uncontaminated sample (calibration sample) and relative resonance frequency shift of the contaminated sample spectral lines. Resonance frequency measurement is based on the $\mathrm{S}_{11}$ minimum detection, which poses certain requirements of the microwave receiver sensitivity. To understand these requirements better, one can derive an expression for the $S_{11}$ parameter magnitude as a function of frequency $f$ in the vicinity of the resonance frequency point $f_{0}$,

$$
\left|S_{11}(\Delta f)\right|=\left|S_{11 \min }\right| \sqrt{1+4 Q^{2} \frac{\Delta f^{2}}{f_{0}^{2}}}
$$

Where

$$
\Delta f=\left|f-f_{0}\right|, \Delta f / f_{0}<<1,
$$

and $\left|S_{11_{\text {min }}}\right|$ is a magnitude of the $S_{11}$ parameter at the resonance frequency $f_{0}$. Q is a quality factor of the sensor probe. The derivation of (4) is detailed in Appendix B. It should be stressed that (4) is derived under the general reflection resonance conditions (Appendix B) and is not specific to the particular type of sensors used in this work.

Using Taylor series expansion in (4) for the small parameter $4 Q^{2} \Delta f^{2} / f_{0}^{2}<0.1$, it is possible to find the $\mathrm{S}_{11}$ magnitude variation $\left|\Delta \mathrm{S}_{11}\right|$ between the contaminated and uncontaminated material spectral lines at the frequency $f_{0}+\Delta f$

$$
\left|\Delta S_{11}\right|=\left|S_{11}^{(c)}\left(f_{0}+\Delta f\right)-S_{11}^{(0)}\left(f_{0}+\Delta f\right)\right|
$$

in the following analytical form

$$
\left|\Delta S_{11}\right|=2\left|S_{11 \min }\right|\left(Q \frac{\Delta f}{f_{0}}\right)^{2}
$$

In (5) $f=f_{0}+\Delta f$ is the resonance frequency of the contaminated sample reflection spectral line, $S_{11}^{(c)}(f)$ and $S_{11}^{(0)}(f)$ are the spectral lines of contaminated and uncontaminated samples and it is assumed that $\left|S_{11_{\text {min }}}\right|$ does not appreciably change with a small frequency offset $\Delta f$.

On the other hand, using (3) it is possible to write the contaminant volumetric concentration resolution as

$$
\Delta V_{c}=D \Delta f / f_{0}
$$

where constant $\mathrm{D}$ is given by a relation

$$
D=2\left(A+\varepsilon_{r}^{\prime(h)}\right) /\left(\varepsilon_{r}^{\prime(h)}-\varepsilon_{r}^{\prime(c)}\right)
$$

Equations (6), (7) specify the best achievable theoretical resolution, in the linear detection regime, of the microplastic contaminant concentration in the soil or aquatic environment:

i) to achieve the required resolution $\Delta V_{c}$, the frequency tuning resolution (frequency step) $\Delta f / f_{0}$, of the source $\mathrm{TX}$ and receiver $\mathrm{RX}$ should be finer than $\Delta V_{c} / D$;

ii) for the specified frequency step $\Delta f / f_{0}$, the microwave receiver RX should possess the amplitude sensitivity better than (6).

The required signal-to-noise ratio $S / N$ at the receiver output is related to the sensitivity (6) by an equation [42]-[44]

$$
\left|\Delta S_{11}\right|=k T B S / N
$$

Where $k$ is the Boltzmann's constant, $T$ is the equivalent noise temperature of the receiver referenced to its input and $B$ is the RX bandwidth, $\left|\Delta S_{11}\right|$ dimension can be converted to Watts assuming $50 \Omega$ terminal impedance [33], [45].

The typical frequency tuning resolution of the phase-lockedloop microwave transmitters and receivers [46], [47] is in the range $10^{-5}-10^{-8}$, which indicates that the theoretical microwave spectroscopic resolution of microplastic concentrations in the environment at tens of ppm level is possible, considering microwave circuitry frequency tuning characteristics.

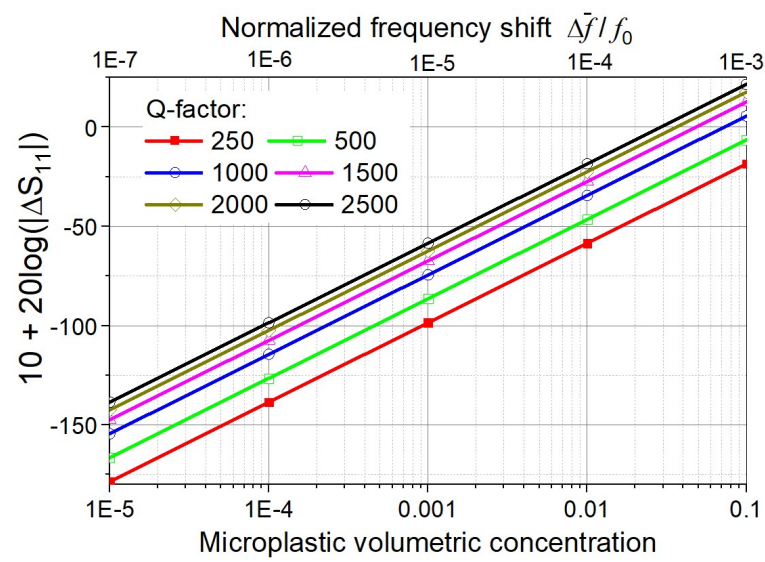

Fig.2. Required receiver sensitivity defined as $10+20 \log \left(\left|\Delta S_{11}\right|\right)$, [45], as a function of microplastic-in-soil volumetric concentration (per unit volume).

Fig.2. shows the required RX sensitivity for the microplasticin-sand detection using the model (2)-(7). The equation parameters in (2), $\varepsilon_{r}^{\prime(h)}=5.60, \varepsilon_{r}^{\prime(c)}=2.59, A=55.8$ are obtained from the experimental results in Section III. The Qfactor varies from 250 (sensor in this work) to 2500. From Fig.2 it can be seen that higher $Q$ factors of the sensing probe allow better contaminant resolution, however even for $Q=2500$, the concentration resolution is limited to $100 \mathrm{ppm}$ (at the $\mathrm{RX}$ sensitivity level $-100 \mathrm{dBm}$ ). Several strategies can be employed to increase the contaminant concentration resolution, at given receiver sensitivity level. These solutions include low-noise signal amplification [19], [20] and/or sensing volume reduction 
[48], thus effectively increasing contaminant volumetric concentration.

\section{EXPERIMENTAL RESULTS}

In this section, experimental data demonstrating microplastic detection and quantification in soil and fresh and salty water are presented. The soil and water samples were artificially contaminated with large concentrations of microplastic, at tens to hundreds of parts-per-thousand (ppt) levels, to achieve accurate experimental control of the contaminant concentration levels in the host material.

\section{A. Experimental Detection and Quantification of Microplastic in Soil}

\section{Soil samples}

Two types of clean soil were prepared: topsoil with high (approximately 20\%) percent of organic matter ( $\mathrm{pH}$ is around $7 \%$ ) and bulk density of $1.33 \mathrm{~g} / \mathrm{cm}^{3}$ and sandy soil with high (more than 90\%) of medium-coarse sand content, bulk density $2.56 \mathrm{~g} / \mathrm{cm}^{3}$. The topsoil and sandy soil samples were collected from the field around the GPS locations $(54.556745,-5.928940)$ and $(54.337611,-5.837946)$, respectively, in Northern Ireland and dried in the oven at $90^{\circ} \mathrm{C}$ for 20 minutes. The topsoil samples were further manually sieved thorough a $0.5 \mathrm{~mm}$ mesh to remove large organic debris and larger particles to homogenize the soil. Both samples are shown in Fig.3a),b).

\section{Microplastic samples}

The microplastic is Nylon 11 (Rilsan (R) powder, with density of $1.03 \mathrm{~g} / \mathrm{cm}^{3}$, Fig. $3 \mathrm{c}$ ). The microplastic powder particle size range is approximately $50 \mu \mathrm{m}-0.5 \mathrm{~mm}$. The complete mechanical and dielectric properties are available elsewhere [49] for the bulk Nylon 11 material. This microplastic is chosen due to its global production volume, and wide applications area, especially in 3D printing [50].

The samples complex permittivity was measured in the 5.0$6.0 \mathrm{GHz}$ band using the conventional free-space measurement method [34]. The measured permittivity values are summarised in Table II for the frequency $5.5 \mathrm{GHz}$.

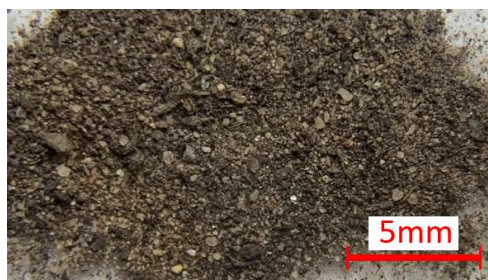

(a)

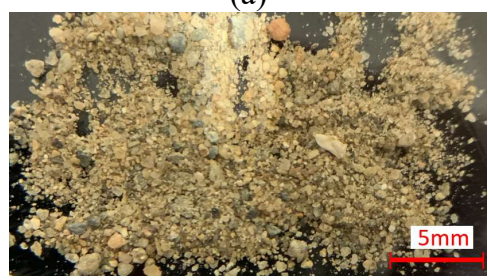

(b)

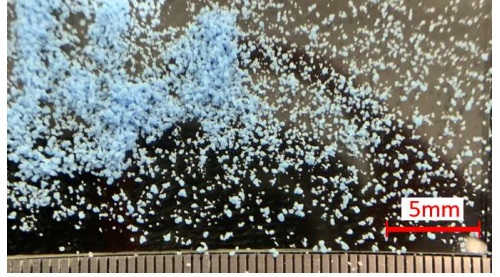

(c)

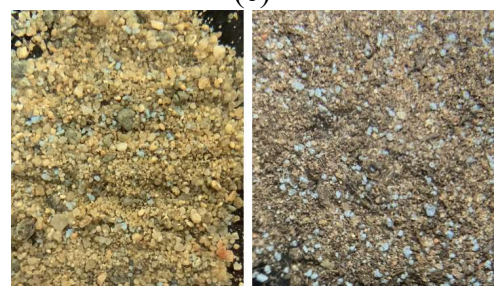

(d)

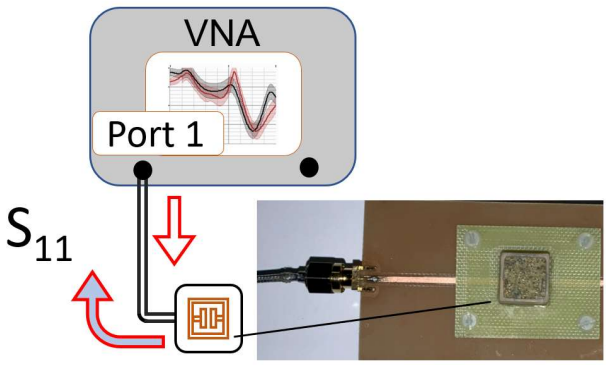

(e)

Fig.3. Samples photographs and schematic diagram of the measurement setup. Samples: (a) topsoil, (b) sandy soil, (c) Nylon 11 powder, (d) microplastic-contaminated samples: left- 10ppt microplasticin-sand, right - 50ppt, microplastic-in-soil. (e) Diagram of the measurement setup.

TABLE II

MeAsured PeRmitTIVITY of DRY SOIL AND NyLON 11 PoWder AT $5.5 \mathrm{GHz}$

\begin{tabular}{ccc} 
& $5.5 \mathrm{GHz}$ & \\
\hline \hline & $\begin{array}{c}\text { Permittivity } \\
\text { real part }\end{array}$ & Loss tangent \\
\hline Topsoil & $4.70 \pm 0.20$ & $0.03 \pm 0.002$ \\
Sandy soil & $5.60 \pm 0.20$ & $0.007 \pm 0.002$ \\
Nylon 11 powder & $2.59 \pm 0.20$ & $0.008 \pm 0.002$ \\
\hline \hline
\end{tabular}

The experimental $\left|\mathrm{S}_{11}\right|$ spectra of the microplasticcontaminated samples are shown in Fig. 4.

The experimental microwave reflectometry procedure is standard and is similar to that described in [18]-[20], [26], [29]. The Keysight E8361C performance network analyzer (PNA) was used to generate the microwave signal in $5 \mathrm{GHz}-6 \mathrm{GHz}$ band and to measure the $\mathrm{S}_{11}$ data. In this experiment, the $\mathrm{PCB}$ resonator aperture dimensions are as follows $d=12 \mathrm{~mm}, L_{c}$ $=8 \mathrm{~mm}, w_{s}=w_{\mathrm{c}}=1 \mathrm{~mm}, g=1.5 \mathrm{~mm}$. The sensor is integrated with an acrylic sample holder of $12 \mathrm{mmx} 12 \mathrm{~mm} \times 10 \mathrm{~mm}$ dimensions and wall thickness $1 \mathrm{~mm}$. The sensor aperture is capacitively fed with a microstrip feeding line with dimensions $L_{\text {feed }}=40 \mathrm{~mm}$, $w_{\text {feed }}=1.5 \mathrm{~mm}$ through an SMA edge connector. The vertical separation between the PCB sensor aperture and the microstrip feeding line is $0.8 \mathrm{~mm}$. PNA input power is $-10 \mathrm{dBm}$. The soil sample was placed in the acrylic sample holder, in direct contact with the sensor aperture.

It was found that the resonance frequency shift is sensitive (up to $\pm 3 \mathrm{MHz}$ ) to the sample particles distribution in the contact with the sensor aperture, which can be explained by a non- 
uniform spatial distribution of the $E$-field, Fig.1b). To achieve accurate sample characterization, ten sub-samples of each microplastic concentration from 0 to $500 \mathrm{ppt}$ with $50 \mathrm{ppt}$ step were prepared. These sub-samples were thoroughly mixed and $\mathrm{S}_{11}$ measured for each sub-sample resulting in the resonance frequency sub-bands $\left(f_{\min , i}, f_{\max , i}\right)$, where index $i=1,2, . ., 10$, corresponds to 50ppt concentration step. Each $\mathrm{S}_{11}$ spectral line shown in Fig. 4 a), b) corresponds to the center of the resonance frequency sub-band. Effectively, this measurement procedure is equivalent to spatial averaging of the contaminated samples particle distribution and the sensor $E$-field non-uniform spatial distribution.

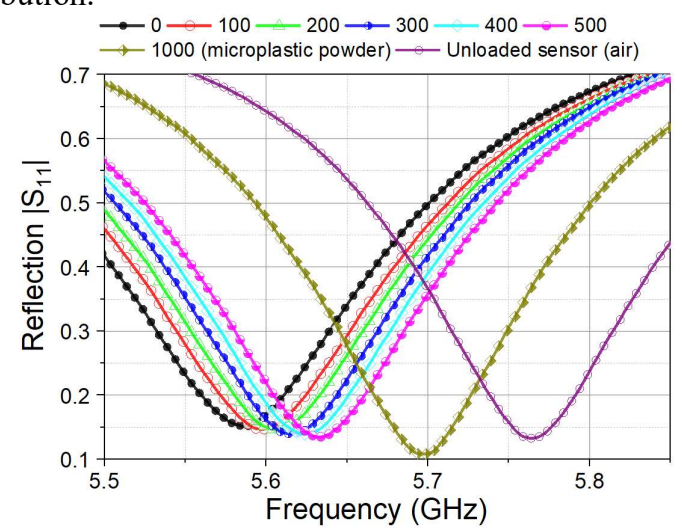

(a)

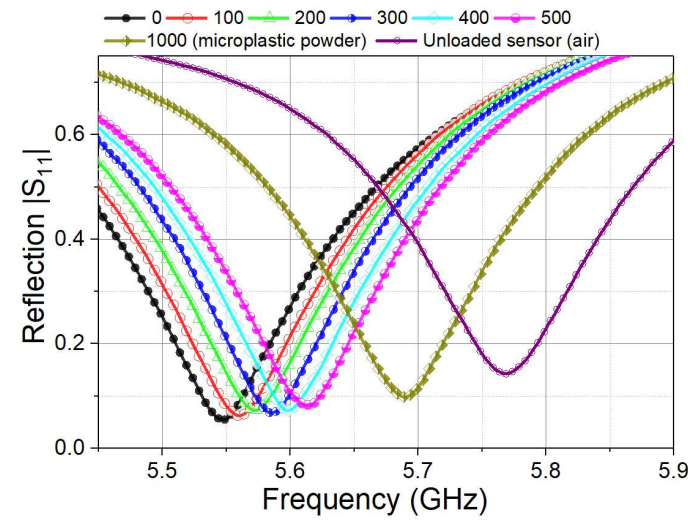

(b)

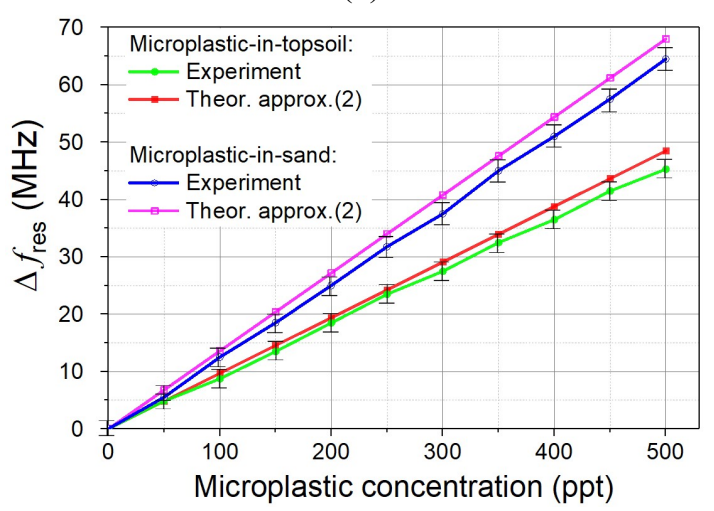

(c)

Fig.4. The measured $S_{11}$ spectra of the microplastic-contaminated samples. (a) Topsoil; (b) contaminated sandy soil. (c) $S_{11}$ resonance frequency shift vs microplastic concentration.

The measured Q-factors of the empty and sample-loaded sensors are summarized in Table III. These Q-factor values can be explained using the permittivity data, Table II which shows that sandy soil has the largest real-part permittivity and the lowest microwave loss. The lower Q-factor of the topsoilloaded sensor is caused by the higher $\tan \delta$ of the topsoil sample.

TABLE III

Q-FACTORS OF EMPTY AND LOADED SENSORS

\begin{tabular}{lc}
\hline \multicolumn{1}{c}{ Sample } & Q-factor \\
\hline Air & 119.4 \\
Uncontaminated sandy soil & 259.8 \\
Uncontaminated topsoil & 92.4 \\
Nylon 11 powder & 157.4 \\
\hline \hline
\end{tabular}

Finally, constant $A$ appearing in (2), (3) was obtained from the measurement data, Fig. 4a), b) by comparing the unloaded and loaded sensor resonance frequency, $A=55.8$.

Fig. 4c) shows the measured resonance frequency shift vs microplastic concentration in topsoil and sand samples and the resonance frequency shift given by a linearized equation (2). The error bars show the resonance frequency sub-bands $\left(f_{\min , i}\right.$, $\left.f_{\max , i}\right)$ variation. It can be seen that the linear approximation (2) is in a good agreement with the experimental data, especially at a lower concentration of the microplastic contaminant in soil.

\section{B. Experimental Detection and Quantification of Microplastic in Water}

In the microwave frequency band $1 \mathrm{GHz}-10 \mathrm{GHz}$, the permittivity and microwave loss contrast between water and most plastics differ by more than an order of magnitude. Specifically, at $5.5 \mathrm{GHz}$ the permittivity and loss tangent of fresh and sea (30ppt) water obtained from the literature [51]-[53] are listed in Table IV suggesting that there is more than 20 times contrast in the real part of permittivity and two orders of magnitude contrast in loss tangent between water and Nylon 11 microplastic powder.

TABLE IV

COMPLEX Relative PERMitTIVITY OF WATER @5.5GHz AND $20^{\circ} \mathrm{C}$

\begin{tabular}{lcc}
\hline \hline Sample & $\operatorname{Re} \varepsilon_{\mathrm{r}}$ & $\begin{array}{c}\text { Loss } \\
\text { tangent }\end{array}$ \\
\hline Freshwater & $72.0 \pm 0.50$ & $0.28 \pm 0.02$ \\
Salty water & $66.0 \pm 0.40$ & $0.80 \pm 0.07$ \\
\hline \hline
\end{tabular}

Sensing in the aquatic environment has several distinct features, dictated by the high permittivity and microwave loss of water [26], [28], [32], [54], [55]. In this study, it was experimentally found that full immersion of the microwave sensor into tap or salty water leads to a considerable reduction of the Q-factor (5 times or more). To maintain high Q essential for accurate contaminant detection, the inductive-capacitive squarering sensor is partially loaded with a water mini-channel of $1 \mathrm{~mm}$ diameter and 9mm length, Fig. 5a), drilled in a miniature acrylic block. This water-filled mini-channel is positioned between the capacitive plates of the sensor, as shown in Fig.5a). Partial loading of the resonator allows to preserve high $\mathrm{Q}$ factor: the measured Q-factors of fabricated sensors are $\mathrm{Q}_{\mathrm{air}}=218.7$ for the sensor with integrated empty acrylic cavity, $Q_{\text {tap_water }}=110.0$ for the sensor with a channel filled with uncontaminated tap water, $Q_{\text {salty_water }}=101.5$ for the sensor loaded with uncontaminated salty water. These data show that Q factor is reduced by two times as compared to the Q-factor of the sensor loaded with the low-loss sand samples. 


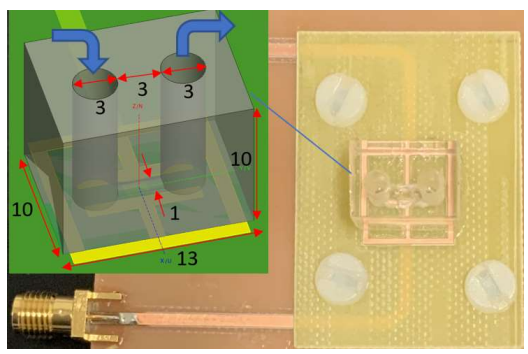

(a)

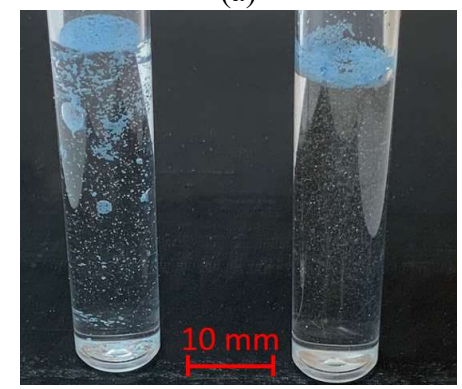

(b)

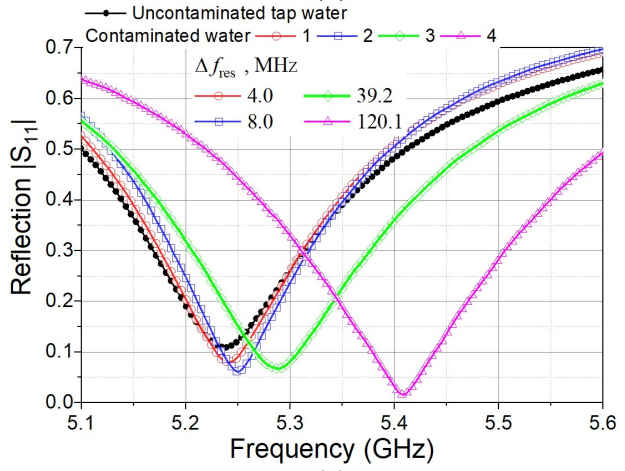

(c)

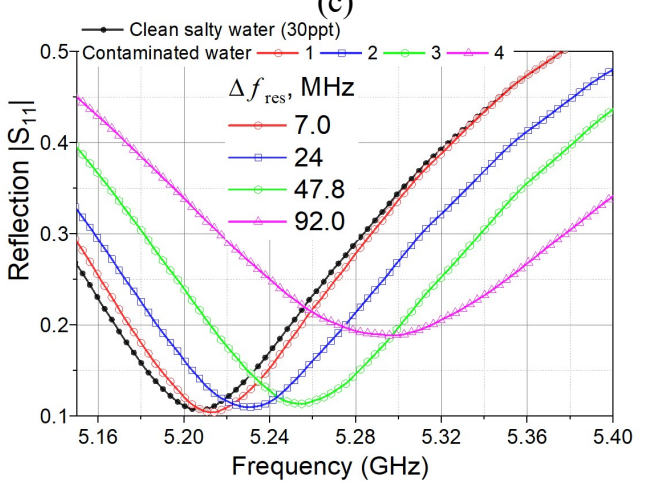

(d)

Fig.5. (a) Square loop capacitive resonator, partially-loaded with water-filled channel, inset shows cavity dimensions in $\mathrm{mm}$. The water flow is streamed inside the acrylic cavity channel between the capacitive plates of the resonator (b) Photograph of the microplastic-water mixture, left- tap water, right- salty water. Measured S11 spectra of the microplastic-contaminated tap, (c) and salty, (d), water.

Another essential feature of microplastic-in-water detection is related to the properties of microplastic-in-water mixture, Fig.5b). Nylon 11 is a hydrophobic material (water absorption index $0.8 \%$ over 24 hours), so it is resistant to solubility with water. In the samples shown in Fig.5b), 50ppt volume fraction of Nylon 11 powder is mixed with tap (left) and salty (right) water. In tap water, microplastic forms an emulsion-like mixture of suspended particles (size is less than $100 \mu \mathrm{m}$ ) and particle mini-clusters of $0.25 \mathrm{~mm}-2 \mathrm{~mm}$ average size. In salty water, the Nylon particle clusters are formed only on the water surface, Fig. 5b) while the distribution of microplastic particles inside the water volume is more uniform than in the fresh water mixture. Since microplastic particles size distribution can vary from approximately $50 \mu \mathrm{m}$ to $0.5 \mathrm{~mm}$, accurate quantification of the $S_{11}$ spectral lines vs particle size distribution requires microsystem approach to microplastic separation in water, such as mesh or membrane filters [23]. The realization of the selfcalibrated sensors based on microplastic particles separation in microfluidic channels will be reported in future work.

In this study, preliminary experimental results demonstrating characteristic resonance frequency variation in the microplastic-contaminated samples are presented in Fig. 5c),d). The overall range of resonance frequency shift was more than $140 \mathrm{MHz}$, depending on the Nylon 11 particle distribution in the water mini-channel. The water flow was controlled by a syringe pump, particle size distribution was monitored under the microscope and $\mathrm{S}_{11}$ measured for specific particle distribution in the fluid mini-channel. In Fig. 5c) the approximate linear size of Nylon 11 mini-clusters in the channel: line $1-0.2 \mathrm{~mm}, 2-$ $0.3 \mathrm{~mm}, 3$ - two clusters $\mathrm{x} 0.4 \mathrm{~mm}$, line 4 - clusters $0.5 \mathrm{~mm}$ and $0.6 \mathrm{~mm}$. Fig. $5 \mathrm{~d}$ ) line $1-0.25 \mathrm{~mm}$, line $2-$ two clusters $\times 0.3 \mathrm{~mm}$, line 3- two clusters $0.35 \mathrm{~mm}$ and $0.45 \mathrm{~mm}$, line 4 - two clusters $0.6 \mathrm{~mm}$ and $1 \mathrm{~mm}$. These data show that the resonance frequency shift increases as a quasi-linear function of the volumetric concentration of microplastic in water, Fig.6, which is in a good qualitative agreement with the model (2). More accurate quantitative characterization of the microplastic-in-water sensor performance requires microfluidic channels equipped with microplastic particles separation or size-sorting [23] and stereo-microscopic techniques for precise microplastic particles size recording. It should be noted that the stereo-microscopic particles size recording would only be required at the sensor calibration stage.

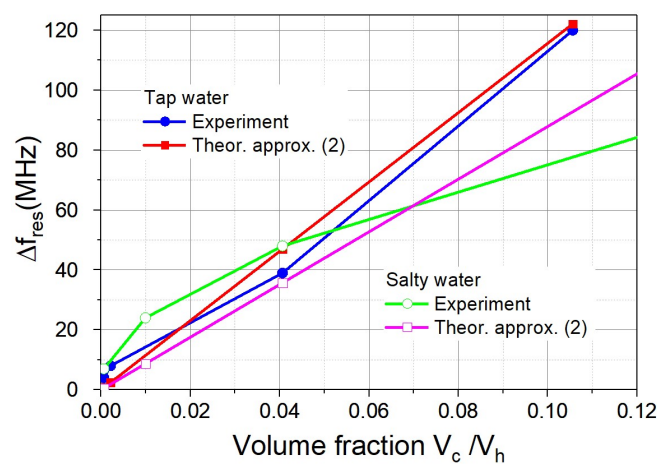

Fig.6. Resonance frequency shift as a function of microplastic volumetric concentration in the acrylic cavity mini-channel filled with a contaminated water sample. Blue dotted line - resonance frequency shift in the tap water samples, Fig.5c). Green hollow dot line - resonance frequency shift in the salty water samples, Fig. $5 d$ ).

\section{CONCLUSIONS}

This study demonstrates that resonance microwave sensing is a promising tool to detect and quantify microplastic in soil and water at the ppt contamination levels. Theoretical model (2) shows that the $\left|\mathrm{S}_{11}\right|$ resonance frequency shift of a microplasticcontaminated sample is a linear function of the microplastic 
volumetric concentration, for low concentrations below 200ppt. Model (2) is in a good agreement with the experimental data at low-ppt microplastic concentration level. Extrapolation of (2) to the sub-ppt microplastic concentration level allows one to estimate the $\left|S_{11}\right|$ resonance frequency shift range for the lower concentration levels of microplastic in soil and water.

Successful detection and quantification of the environmental microplastic at or below the tens-of-ppm level critically depends on the microwave receiver sensitivity, as suggested by the mathematical model (3)-(8). Analysis in Section II shows that microplastic detection at low concentration levels below $100 \mathrm{ppm}$ requires highly sensitive microwave receivers with sensitivity below $-100 \mathrm{dBm}$. This high-sensitivity requirement can be relaxed by using higher Q sensors or by reducing the sensing volume of the sample under test, effectively increasing the volumetric contaminant concentration.

\section{APPENDIX A}

The resonance frequency $f_{L}$ of a microwave sensor loaded with a material sample with real-part relative permittivity $\varepsilon_{r}^{\prime}$, and the resonance frequency $f_{\mathrm{U}}$ of an unloaded sensor (air) are connected by the relation [41]

$$
f_{L}^{2} / f_{U}^{2}=(1+A) /\left(\varepsilon_{r}^{\prime}+A\right)
$$

where $A$ is a constant that depends on the sensor geometry and sample permittivity $\varepsilon_{r}^{\prime}$. If the host medium (soil or water, ${\varepsilon_{r}^{\prime}}_{r}^{(h)}$ ) is contaminated with microplastic $\left(\varepsilon_{r}^{\prime(c)}\right)$ such that the permittivity of the mixture

$$
\varepsilon_{r}^{\prime}=\varepsilon_{r}^{\prime(h)}-\Delta \varepsilon
$$

where $\Delta \varepsilon<<\varepsilon_{r}^{\prime(h)}$ is a small permittivity variation,

$$
\Delta \varepsilon=\varepsilon_{r}^{\prime(h)}-\left(V_{h} \varepsilon_{r}^{\prime(h)}+V_{c} \varepsilon_{r}^{(c)}\right)=V_{c}\left(\varepsilon_{r}^{\prime(h)}-\varepsilon_{r}^{\prime(c)}\right)
$$

one can write the expression for the resonance frequency of the sensor loaded with a contaminated sample as

$$
f_{L}=\frac{f_{U} \sqrt{1+A}}{\sqrt{A+\varepsilon^{\prime}(h)}-\Delta \varepsilon}=\frac{f_{U} \sqrt{1+A}}{\sqrt{A+\varepsilon_{r}^{\prime}(h)} \sqrt{1-\frac{\Delta \varepsilon}{A+\varepsilon_{r}^{\prime}(h)}}}
$$

Taking into account that

$$
\Delta \varepsilon /\left(A+\varepsilon_{r}^{\prime(h)}\right)<0.1
$$

and using Taylor expansion for inverse square root in (A4), one can derive the equation for the resonance frequency $f_{L}$ of the sample with microplastic contaminant volumetric fraction $V_{c}$,

$$
f_{L}=f_{0}+\frac{1}{2} f_{0} \frac{V_{c}\left(\varepsilon_{r}^{\prime(h)}-\varepsilon_{r}^{\prime(c)}\right)}{A+\varepsilon_{r}^{\prime(h)}}
$$

where $f_{0}$ is the resonance frequency of the sensor loaded with an uncontaminated sample, $V_{c}=0$. Equation (2) follows from (A6).

\section{APPENDIX B}

The magnitude of the $S_{11}$ parameter of a resonance sensor can be written in a general form [33] as

$$
\left|S_{11}\right|=\left|\left(Z_{L}-Z_{0}\right) /\left(Z_{L}+Z_{0}\right)\right|
$$

where $Z_{L}=R+j\left(\omega^{2} L C-1\right) / \omega C$ is a complex impedance of a resonance sensor, $R, L, C$ are the equivalent lumped circuit parameters of the sensor, $Z_{0}$ is an impedance of the sample material, [33]. In the proximity to the resonance, $f=f_{0} \pm \Delta f$, the real part of the denominator in (B1) dominates over the imaginary part and is almost constant; the numerator in (B1) can be represented in the form

$$
Z_{L}-Z_{0}=\operatorname{Re} \Delta Z+j L\left(\omega^{2}-\omega_{0}^{2}\right) / \omega
$$

Expanding $\omega^{2}=4 \pi^{2}\left(f_{0}+\Delta f\right)^{2}$, neglecting higher-order terms $\sim \Delta f^{2}$ and taking the module of (B2), one can derive the approximation for the $\left|\mathrm{S}_{11}\right|$ magnitude around the resonance frequency $f=f_{0}+\Delta f$,

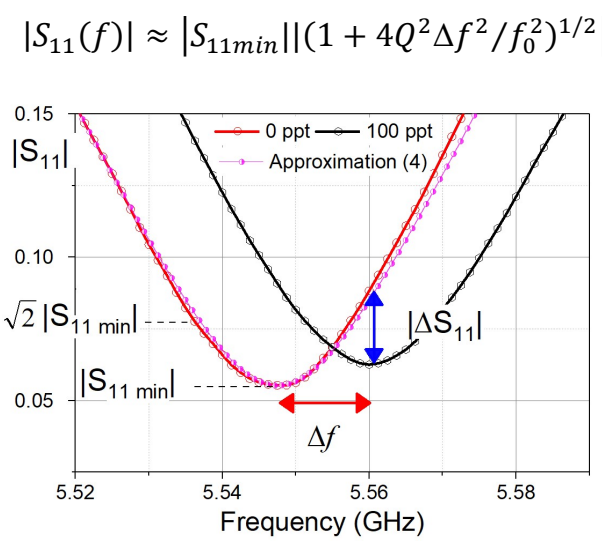

Fig A1. Reflection parameter $\left|S_{11}\right|$ : red dotted line - measurement, magenta solid line- approximation. The approximation (B3) is in excellent agreement with the measured $\left|S_{11}\right|$ data.

\section{ACKNOWLEDGMENT}

The author would like to thank anonymous Reviewers for insightful comments and suggestions that allowed to improve the original manuscript.

\section{REFERENCES}

[1] A.L. Andrady, "Microplastics in the marine environment," Mar. Pollut. Bull. vol. 62, no. 8, pp. 1596-1605, 2011.

[2] K. Cox, A. Garth, G. Covernton, et al., "Human Consumption of Microplastics," Environ. Sci. Technol. vol. 53, no. 12, pp.7068-7074, 2019.

[3] J.P. Costa J.P, P. Santos, A. Duarte, T. Rocha-Santos, "(Nano)plastics in the environment - Sources, fates and effects,” Sci. Total Environ., 566-567, pp. 15-26, 2016.

[4] L. Hernandez, E.G. Xu, H. Larsson, et al., "Plastic Teabags Release Billions of Microparticles and Nanoparticles into Tea," Environ. Sci. Technol. vol. 53, no 21, pp. 12300-12310, 2019.

[5] R. C. Thompson, Y. Olsen, R.P. Mitchell, et. al. "Lost at sea: Where is all the plastic?" Science, vol. 304 (5672), pp. 838838,2004 . 
[6] R. Geyer, J. Jambeck, K. Lavender Law, "Production, use, and fate of all plastics ever made," Science Advances, vol. 3, no. 7, 2017.

[7] Parker, L. A whopping 91\% of plastic isn't recycled, National Geographic Dec. 2018, available online https://www.nationalgeographic.com/news/2017/07/plasticproduced-recycling-waste-ocean-trash-debris-environment/

[8] Keller, J. Jimenez-Martinez, D. Mitrano, "Transport of Nano- and Microplastic through Unsaturated Porous Media from Sewage Sludge Application," Environ. Sci. Technol. vol. 54, no. 2, pp. 911-920, 2020. DOI: 10.1021/acs.est.9b06483

[9] Boots, C.W. Russell, D. Green, "Effects of Microplastics in Soil Ecosystems: Above and Below Ground," Environ. Sci Technol. vol. 53, no. 19, pp. 11496-11506, 2019.

[10] C.M. Rochman, M.A. Browne, B. Halpern, et.al., "Classify plastic waste as hazardous," Nature 494, pp. 169-171, 2013.

H. Bouwmeester, P. Hollman, R. Peters, "Potential Health Impact of Environmentally Released Micro- and Nanoplastics in the Human Food Production Chain: Experiences from Nanotoxicology," Environ Sci Technol., vol. 49 , no. $15,8932-8947,2015$

[12] V. Hidalgo-Ruz, L. Gutow, R. Thompson, M. Thiel, "Microplastics in the Marine Environment: A Review of the Methods Used for Identification and Quantification,' Environ. Sci. Technol. vol. 46, no. 6, pp. 3060-3075, 2012.

[13] J. Sun, X. Dai, Q. Wang, et al. "Microplastics in wastewater treatment plants: Detection, occurrence and removal," Water Research, vol. 152, pp. 21-37. 2019.

[14] J. Möller, M. Löder, C. Laforsch, "Finding Microplastics in Soils: A Review of Analytical Methods," Environ. Sci. Technol. vol. 54, no. 4, pp. 2078-2090, 2020.

[15] Y.K. Song, S.H. Hong, M. Jang, et al. "A comparison of microscopic and spectroscopic identification methods for analysis of microplastics in environmental samples," Mar. Pollut. Bull., vol. 93, pp. 202-209, 2015.

[16] T. Rocha-Santos, A.C. Duarte, "A Critical Overview of the Analytical Approaches to the Occurrence, the Fate and the Behavior of Microplastics in the Environment," Trends Anal. Chem. vol. 65, pp. 47-53, 2015.

[17] H. Ball, R. Cross, E. Grove, et al., "Sink to River - River to Tap. A Review of Potential Risks from Nanoparticles and Microplastics". Report 19/EQ/01/18, UK Water Industry Research Limited 2019, ISBN 978-1-84057-874-4.

[18] O. Malyuskin, V. Fusco, "High-Resolution Microwave NearField Surface Imaging Using Resonance Probes," IEEE Trans. Instrum. Measurement, vol. 65, no. 1, pp. 189-200, 2016.

[19] R. Mirzavand, M. Mahdi Honari, P. Mousavi, "HighResolution Dielectric Sensor Based on Injection-Locked Oscillators," IEEE Sensor Journ., vol. 18, no. 1, pp. 141-148, 2018.

[20] G. Vlachogiannakis, Z. Hu, H. Shivamurthy, et al., "Miniaturized Broadband Microwave Permittivity Sensing for Biomedical Applications," IEEE Journal Electromagnetics, $R F$ and Microwaves in Medicine Biol. vol. 3, no. 1, pp. 48-55, 2019.

[21] J. Aguzzi, D. Chatzievangelou, S. Marini, et al., "New HighTech Flexible Networks for the Monitoring of Deep-Sea Ecosystems," Environ. Sci. Technol., vol. 53, no. 12, pp. 6616-6631, 2019

[22] J. Sun, X. Dai, Q. Wang, et al. "Microplastics in wastewater treatment plants: Detection, occurrence and removal," Water Research, vol. 152, pp. 21-37. 2019.

[23] B. Nguyen, et. al., "Separation and Analysis of Microplastics and Nanoplastics in Complex Environmental Samples,' Accounts of Chemical Research, vol. 52, no. 4, pp. 858-866, 2019.

[24] F. Corradini et.al., "Predicting soil microplastic concentration using vis-NIR spectroscopy," Science Tot. Environment, vol.650, part 1, pp. 922-932, 2019.

[25] Dielectric Resonators, Second Ed. D. Kajfez and P. Guillon, Noble 1998.

[26] B. D. Wiltshire and M. H. Zarifi, "3-D Printing Microfluidic Channels with Embedded Planar Microwave Resonators fo
RFID and Liquid Detection," IEEE Microwave Wireless Comp. Lett., vol. 29, no. 1, pp. 65-67, Jan. 2019.

[27] H. Hallil et al., "Differential Passive Microwave Planar Resonator- Based Sensor for Chemical Particle Detection in Polluted Environments," IEEE Sensors Journal, vol. 19, no. 4, pp. 1346-1353, 2019.

[28] Z. Abedeen, P. Agarwal, "Microwave sensing technique based label-free and real-time planar glucose analyzer fabricated on FR4," Sensors and Actuators A, vol. 279, pp. $132-139,2018$

[29] A. Chahadih et al., "Microwave/microfluidic sensor fabricated on a flexible Kapton substrate for complex permittivity characterization of liquids," Sensors and Actuators A, vol. 229, pp. 128-135, 2015.

[30] I. Prayudi, M. Abou-Khousa, A. Al-Durra and K. AlWahedi, "RF Sensor for Material Delivery Monitoring in Powder Feeding Systems," IEEE Sensors Journal, vol. 16, no. 23, pp. 8434-8439, 2016.

[31] F. Artis et al., "Microwaving Biological Cells: Intracellular Analysis with Microwave Dielectric Spectroscopy," IEEE Microwave Magazine, vol. 16, no. 4, pp. 87-96, May 2015.

[32] M. H. Zafiri, M. Daneshmand, "Liquid sensing in aquatic environment using high quality planar microwave resonator," Sensors and Actuators B, vol. 225, pp. 517-521, 2016.

[33] D. M. Pozar. Microwave Engineering, John Wiley \& Sons, 2011.

[34] K.C. Yaw, Measurement of Dielectric Material Properties. Appl. Note 04.2012-RAC0607-0019-1-4E. Rohde \& Schwarz, 2012. Available online: https://cdn.rohdeschwarz.com/pws/dl downloads/dl application/00aps unde fined/RAC-0607-0019 1 5E.pdf

[35] B. D. Wiltshire and M. H. Zarifi, "3-D Printing Microfluidic Channels with Embedded Planar Microwave Resonators for RFID and Liquid Detection," IEEE Microwave Wireless Comp. Lett., vol. 29, no. 1, pp. 65-67, Jan. 2019.

[36] G. Galindo-Romera, F. Javier Herraiz-Martínez, M. Gil, et.al., "Submersible Printed Split-Ring Resonator-Based Sensor for Thin-Film Detection and Permittivity Characterization," IEEE Sensors Journal, vol. 16, no. 10, pp. 3587-3596, 2016.

[37] M. A. Karimi, M. Arsalan and A. Shamim, "Low Cost and Pipe Conformable Microwave-Based Water-Cut Sensor," IEEE Sensors Journal, vol. 16, no. 21, pp. 7636-7645, Nov.1, 2016.

[38] S. Dey, J. K. Saha and N. C. Karmakar, "Smart Sensing: Chipless RFID Solutions for the Internet of Everything," IEEE Microwave Magazine, vol. 16, no. 10, pp. 26-39, Nov. 2015.

[39] T. C. Choy, Effective Medium Theory, Principles and Applications. Oxford Science Publications, Oxford, 1998.

[40] R. Esquivel-Sirvent, G. C. Schatz, "Mixing rules and the Casimir force between composite systems," Phys. Rev. A., vol. 83, 042512, 2011.

[41] O. Malyuskin, et.al., "Characterization of microwave absorption in carbon nanotubes using resonance aperture transmission method", submitted to Journ. Applied Phys., 2020, available: arXiv:2001.09184

[42] M. J. Usher and D.A. Keating, Sensors and Transducers Characteristics, Applications, Instrumentation, Interfacing. MacMillan Press, London, 1996.

[43] Fundamentals of RF and Microwave Noise Figure Measurements, Application note, Keysight. Available online: https://www.keysight.com/gb/en/assets/701806808/application-notes/5952-8255.pdf

[44] G. A. Leonov, et al., "Analytical method for computation of phase-detector characteristic," IEEE Trans. Circuits Syst. II: Express Briefs, vol. 59, no. 10, pp. 633-637, 2012.

[45] Voltage to Power Conversion in a $50 \Omega$ System, technical note,

https://www.rfcafe.com/references/electrical/pwr2volts.htm

[46] I. Bashir, R. Staszewski, P. Balsara, "A Digitally Controlled Injection-Locked Oscillator with Fine Frequency Resolution," IEEE Journ. Solid-state Circuits, vol. 51, no. 6, pp. 1347-1360, 2016. 
[47] L. Fanori, A. Liscidini, R. Castello, "3.3GHz DCO with a frequency resolution of $150 \mathrm{~Hz}$ for All-digital PLL," - IEEE International Solid-State Circuits Conference, 2010. DOI: 10.1109/ISSCC.2010.5434057

[48] J. Leroya, C. Dalmaya, A. Landoulsi, A., et. al., "Microfluidic biosensors for microwave dielectric spectroscopy," Sensors and Actuators A: Physical, vol. 229 pp. 172-181, 2015.

[49] Polyamide - Nylon 11 material information datasheet, Available online: www.goodfellow.com/E/PolyamideNylon-11.html

[50] PA11 vs PA12: 3D printing of performance polymers, Arkema technical note, Available online: https://www.arkema.com/en/webzine/story/PA11-vs-PA123D-printing-of-performance-polymers/

[51] Chaplin, M.; Water Structure and Science, Water and Microwaves Available online: http://www1.lsbu.ac.uk/water/microwave water.html

[52] J. Wang, A. Nguyen, "Historical perspective A review on data and predictions of water dielectric spectra for calculations of van der Waals surface forces," Advances in Colloid and Interface Science, vol. 250, pp. 54-63, 2017.

[53] W.J. Ellison, "Permittivity of pure water, at standard atmospheric pressure, over the frequency range $0-25 \mathrm{THz}$ and the temperature range $0-100{ }^{\circ} \mathrm{C}, "$ Journal Physical Chemistry Reference Data, vol. 36, pp. 1-18, 2007.

[54] Smart Sensors for Real-Time Water Quality Monitoring, Ed. S. Mukhopadhyay, A. Mason. Springer 2013.

[55] S. Mohammadi A. Nadaraja, D. Roberts, et al., Real-time and hazard-free water quality monitoring based on microwave planar resonator sensor, Sensors and Actuators A: Physical, vol. 303, no. 1, 111663, 2020.

[56] B. Nguyen et al., "Separation and Analysis of Microplastics and Nanoplastics in Complex Environmental Samples," Acc. Chem. Res., vol. 52, pp. 858-866, 2019.

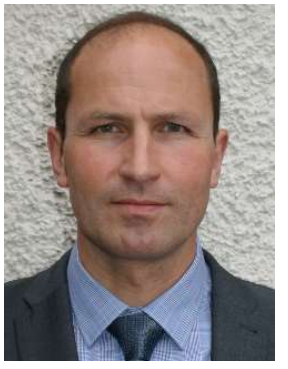

Oleksandr Malyuskin is a Senior Lecturer (Associate Professor) at the Centre for Wireless Innovation, ECIT, Queen's University Belfast. His research interests are focused on microwave imaging and sensing for industrial, environmental and biological applications, advanced wireless communications including time-reversal, retrodirective and electricallysmall antenna arrays, electromagnetic metasurfaces and metamaterials. $\mathrm{He}$ is currently involved, as a leading researcher in a number of projects aimed at the nanomaterials and biomaterials characterization and nanomaterials-based sensing. He has co-authored more than 50 IEEE publications in the field of microwave imaging, sensing and wireless communications. 